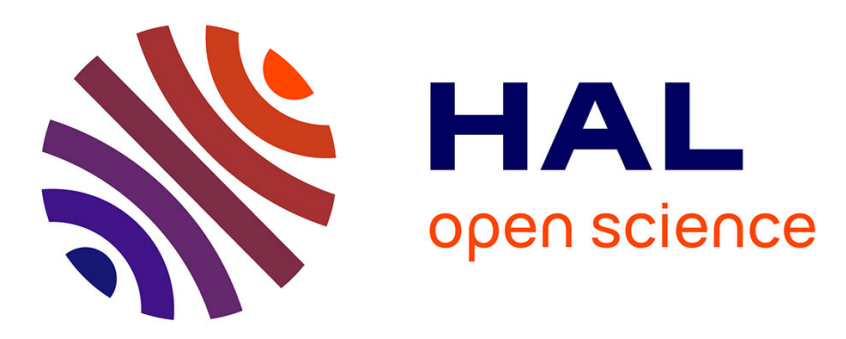

\title{
Stochastic models of systems for Nanotechnology: from micro to macro scale
}

\author{
Savino Longo, Gaia Micca Longo, Khaled Hassouni, Armelle Michau, \\ Swaminathan Prasanna
}

\section{To cite this version:}

Savino Longo, Gaia Micca Longo, Khaled Hassouni, Armelle Michau, Swaminathan Prasanna. Stochastic models of systems for Nanotechnology: from micro to macro scale. 2020. hal-03047915

\section{HAL Id: hal-03047915 https://hal.science/hal-03047915}

Preprint submitted on 9 Dec 2020

HAL is a multi-disciplinary open access archive for the deposit and dissemination of scientific research documents, whether they are published or not. The documents may come from teaching and research institutions in France or abroad, or from public or private research centers.
L'archive ouverte pluridisciplinaire HAL, est destinée au dépôt et à la diffusion de documents scientifiques de niveau recherche, publiés ou non, émanant des établissements d'enseignement et de recherche français ou étrangers, des laboratoires publics ou privés. 


\title{
Stochastic models of systems for Nanotechnology: from micro to macro scale
}

\author{
Savino LONGO ${ }^{1,2}$, Gaia MICCA LONGO ${ }^{1,2}$, Khaled HASSOUNI $^{3}$, Armelle MICHAU ${ }^{3}$, \\ Swaminathan PRASANNA ${ }^{3}$
}

${ }^{1}$ Department of Chemistry - Università degli Studi di Bari Aldo Moro - Via Orabona 4 - 70125 Bari, Italy

${ }^{2}$ Istituto per la Scienza e Tecnologia dei Plasmi - Consiglio Nazionale delle Ricerche, Bari Section

- Via Amendola 122/D - 70125 Bari, Italy

${ }^{3}$ Laboratoire des Sciences des Procédés et des Matériaux, UPR3407, CNRS, Universite Paris 13, avenue Jean-Baptiste Clément, 93430 Villetaneuse France

\begin{abstract}
Computer modeling technique based on the theory of stochastic processes have been used in order to provide a realistic simulation of the behavior of nanoscopic systems, related in particular to plasma reactors in microelectronic device production. Basing on decades of experience, we show here, with new results, that the universality of such methods allows the development of codes with the highest reusability and versatility, crossing the barrier of scale. At the smallest scale, the quantum calculation of the potential energy surface and spectroscopic properties of hydrogen species under nanoconfinement conditions display the effects due to the dimension and the symmetry of the confining potential well. Nanoparticles dispersed as aerosol in plasma feature strong fluctuations in temperature and charge which may affect the processing of silicon wafers. At the macroscopic scale, using a stochastic solution of transport equations, it is possible to describe laboratory or industrial systems for the production or treatment of nanomaterials, also exploiting the analogy between neutral particle transport and radiative transfer and information obtained by molecular simulations. These findings are relevant in the control of solid-particle contamination in the manufacture of electronic components and in other fields.
\end{abstract}

\section{Introduction}


Computer models based on the theory of stochastic processes allow to study different systems of interest for Nanotechnology in a quantitative way, and to analyze: the microscopic scale, at the level of single atoms or molecules or ions, taking the quantum effects into account; the mesoscopic scale of objects with dimensions of the order of tens or hundreds of microns, avoiding the detail of the molecular scale; the macroscopic scale, i.e. entire reactors in the treatment of surfaces with special properties and the production of nanostructures on a large scale.

Stochastic systems can be studied by solving one of a limited class of evolution equations for probability.

If $w_{n m}$ are the transitions probabilities between the states $n$ and $m$, the evolution of most system is governed by a set of ordinary differential equation collectively called Master Equation:

$$
\frac{d P_{n}}{d t}=\sum_{m} w_{m n} P_{m}-P_{n} \sum_{m} w_{n m}
$$

where $\operatorname{Pn}(t)$ is the probability distribution function, such that at time $t$ the system is in the state $n$. When the states span on a continuum, $n$ is replaced by dynamic variables like $\boldsymbol{r}$. Such an equation can describe, for example, quantized charge state of a nanoparticle.

As variations of such a continuum formalism, we have the Boltzmann equation, which describe the interaction of classical particles via the related cross sections, and the equations for ray propagations in radiative transfer. When the state variable undergoes small variations in a single microscopic interaction, a partial differential equation, the Fokker-Planck equation, can be applied:

$$
\frac{\partial P(\boldsymbol{r}, t)}{\partial t}=D \nabla^{2} P-\nabla(\boldsymbol{a} . \nabla P)
$$

The diffusion coefficient $D$ accounts for thermal fluctuations, while the vector quantity $\boldsymbol{a}$ represents an advective flow. Such an equation can be applied to the diffusion of particles performing a Brownian motion; with minor variation, it can be applied to calculate the quantum state of a microscopic system. These equations can be solved by techniques based on the so-called realization of the implied stochastic process: this means simulating an ensemble of mathematical dots distributed as $P$, and simulating events on any single dot in order to reproduce the transition probabilities $w$ in the case of equation (1), and the transport coefficients $\boldsymbol{a}$ and $D$ in equation (2). In the case of equation (1), dots (like particles with a given velocity) are randomly displaced with probability $w d t$ in a short time $d t$. In the case of equation (2), the new random position of any dot is determined based on the short-time propagator (Green function): 


$$
G(d \boldsymbol{r}, d t)=(4 \pi D t)^{-3 / 2} \exp \left(-\frac{d(\boldsymbol{r}-\boldsymbol{a} d t)^{2}}{4 D d t}\right)
$$

The most important advantage of the stochastic approach is its universality. Either a random jump process or a continuum one, diffusion process can be considered as the basic feature of many problems in molecular structure, gas/solid interaction, transport phenomena. In many cases, the random processes are realized in the computer code by using random numbers, thereby producing one of an endless varieties of Monte Carlo models. These models can describe the quantum wavefunction of a molecule, the interaction of a nanoparticle with its environment, the motion of atoms and ions in the macroscale.

For many years, the scientific activity of our collaboration has involved the development of calculation programs based on stochastic process theory concepts, in some cases including the development of innovative algorithms. Taking advantage of the positive properties of the stochastic simulation, the application of the simulation programs developed to nanotechnology includes both the microscopic and the macroscopic scale. On a microscopic scale, we are able to study the electronic structure of small molecular systems, in particular connected with hydrogen plasma technology. On a macroscopic scale, we are able to perform simulations of plasma reactors and systems in molecular regime for the production and treatment of nanomaterials at low temperature.

On the Italian side, these researches were carried out within the CNR Institute of Nanotechnology in the course of several years of activity, although they are presently continued in association with the newly created Institute of Plasma Science and Technology.

In this short highlight, we will summarize some of these applications by choosing in particular recent case studies, trying to highlight the characteristics of these research themes that have led to their success. Some new calculations are produced to illustrate the kind of results which can be obtained. We will proceed from a decidedly nanoscopic scale of molecules and ions with a small number of electrons confined in cavities of various kinds, then we will analyze the study of nanometer-sized dust grains that exhibit special properties from a thermal and electrical point of view, and we will arrive at the simulation of systems on the laboratory scale and used for the production of nanomaterials in both fundamental and applicative contexts.

\section{Quantum Monte Carlo models of nano-confined hydrogen species}

Quantum Monte Carlo (QMC) methods are becoming more and more interesting in the determination of the quantum state of nano-systems, as they allow to minimize the use of approximations and analytical methodologies and do not need heavy adaptations concerning geometry changes. For this 
reason, they are very useful in the study of systems confined in potential wells of nanometer size and of different shape. Such simple confined physical systems have indeed a very long tradition in physics, not only as basic quantum systems, but also as models for extreme high-pressure states of matter, spectroscopically active defects in solid lattices and chemical species in molecular cages [Sabin, 2009; Sen, 2014]. In this regard, the confined states of molecular and ionic states of hydrogen, like $\mathrm{H}_{2}{ }^{+}$and $\mathrm{H}_{2}$ have been much studied, since they allow much accurate calculations [Colín-Rodríguez, 2011]. At the same time, these systems have numerous real applications in the fields of nanotechnology, microelectronics, energy management and quantum computing [Harrison, 2005].

Among several Quantum Monte Carlo methods available [Foulkes, 2001], the diffusion Monte Carlo (DMC) method offers the possibility of considering confining walls of any geometry while retaining the use of cartesian coordinates and to include any distribution of positive charge within the cavity. DMC makes use of the similarity between the imaginary time Schrödinger equation and a generalized diffusion equation, which, as shortly discussed in the introduction, can be solved using a stochastic calculus and simulating a random walk. Basically, the diffusion process of numerous points in the phase space, or "walkers" is simulated. The kinetic energy term of the wave equation is connected with the diffusion process, while the potential energy terms formally produce reactions of destruction or multiplication of the walkers, which are consequently included in the stochastic process. The eigenvalue of the energy is adjusted in the course of the calculation until it reaches its exact value, which is reached when the average number of walkers remains constant during the stochastic process, as demonstrated by the theory. The estimated eigenvalue is then increased if the number of walkers is increasing and decreased if the number of particles is decreasing: this simple technique leads to a statistically exact estimate of the ground state energy.

The method always provides the energy of the ground state of the system, with a very limited number of numerical parameters to be optimized and no need to expand the wave function, even if statistical convergence may require significant calculation times. Excited states can be found by looking for the ground state with restrictions in the form of nodal surfaces, that can be often identified a priori thanks to symmetry considerations.

On this basis, in recent years we have developed a suite of Diffusion Monte Carlo codes designed for the study of hydrogen-based and nano species confined by infinite potential wells of various geometries [Micca Longo, 2015a; Micca Longo, 2015b; Micca Longo, 2015c; Longo, 2018]. In contrast with previous Monte Carlo calculations [Sarsa, 2012], our programs do not employ an analytic guess wavefunctions to reduce variance by the importance sampling technique. Instead, it performs very long imaginary time evolutions, while averages are collected. While this technique leads to somewhat larger oscillations and fluctuations compared to the employ of analytical guesses, it has the advantage to be applicable to different confinement geometries with minimal variations, without having to change the expression of the guess wavefunction, since no such guess is used. This technique allows to study different systems, in the ground and excited states, under different geometries of confinements, using essentially the same program. 
We found that the lowest excited states of the $\mathrm{H}_{2}{ }^{+}$molecule (the $\Sigma_{\mathrm{u}}, \Pi_{\mathrm{g}}$ and $\Pi_{\mathrm{u}}$ ones) in spherical confinement conditions have potential energy surfaces (PESs) similar to those of the ground state, even if, without confinement, they are repulsive states (Figure 1, a-b) [Micca Longo, 2015a; Micca Longo, $2015 b]$. The energy reference of any curve in figure $2 b$ is the same and has been chosen in such a way that, asymptotically at $d=+\infty$, the energy of the unconfined system $\mathrm{H}(1 \mathrm{~s})+\mathrm{H}^{+}$is equal to zero. The same reference is used for the other curves: this is why the dissociation limit energy in figure $2 b$ increases when the confinement become tighter. As concerns the electronic ground state, the compression produces an increase of the curvature of the PES at its minimum due to compression of the electron cloud: accordingly, an increase in the Raman frequencies is predicted, which in principle should be detectable in experiments.

A recent result (Figure 1, c-d) is the first calculation of the PESs of the $\mathrm{H}_{2}{ }^{+}$ion confined by an octahedral potential well, which describes the quantum state of this species in the cavities of real crystal lattices much better than a spherical or ellipsoidal potential well [Longo, 2018]. In this case, the PES is quite complex since it depends not only on the internuclear distance but also on the orientation of the molecular axis into the well.
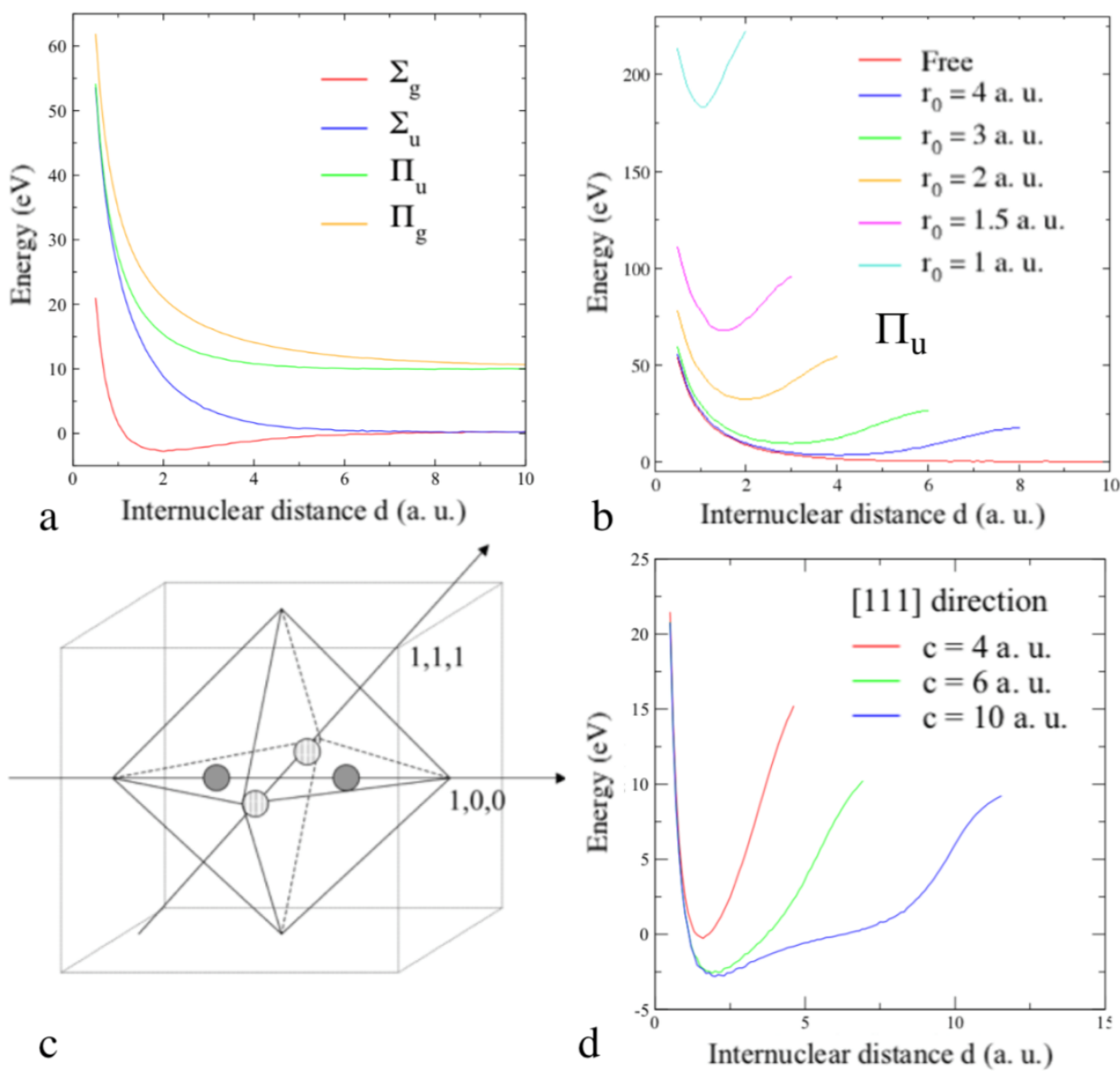
Figure 1. The $\mathrm{H}_{2}{ }^{+}$system with different nanoconfinement geometries studied with the DMC code: a) potential energy surfaces of the unconfined molecule, in its ground state $\Sigma_{g}$ and in its three lowest excited electronic states $\Sigma_{u}, \Pi_{u}, \Pi_{g}$; b) potential energy surfaces of the excited $\Pi_{u}$ state confined into a spherical cavity ( $\mathrm{r}_{0}$ is the cavity radius, a.u. $=$ atomic units): the confinement changes the character of the state of $\Pi_{u}$ symmetry, which develops a pronounced minimum which is absent in the free molecular state.; c) geometry of the tetrahedral confinement of $\mathrm{H}_{2}{ }^{+}$; d) potential energy surfaces of $\mathrm{H}_{2}{ }^{+}$for different degrees of octahedral confinement ( $\mathrm{c}$ is the distance between the center and any vertex of the octahedral hole).

After [Micca Longo, 2015a; Micca Longo, 2015b, Longo 2018].

Another advantage of the DMC method is that, in the case of few-electron systems, quantum correlations are taken into account exactly. Although the results presented in this account refer to a one-electron system, the method can be easily applied to few-electron systems by increasing the dimensionality of the problem: we have recently studied the confinement in different geometries (including nanotubes) of the $\mathrm{H}_{2}$ molecule and the triatomic ion $\mathrm{H}_{3}{ }^{+}$. These calculations are being finalized: preliminary results of the collinear $\mathrm{H}_{3}{ }^{+}$confined in a cylindrical well mimicking a nanotube are shown in figure 2 and 3:

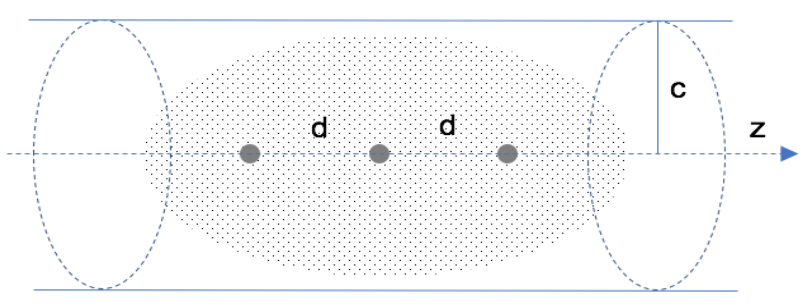

Figure 2. Collinear $\mathrm{H}_{3}{ }^{+}$confined in a longitudinally infinite cylindrical potential well, mimicking a nanotube. The two internuclear distances are equal and they are represented by $d . c$ is the confinement dimension. 


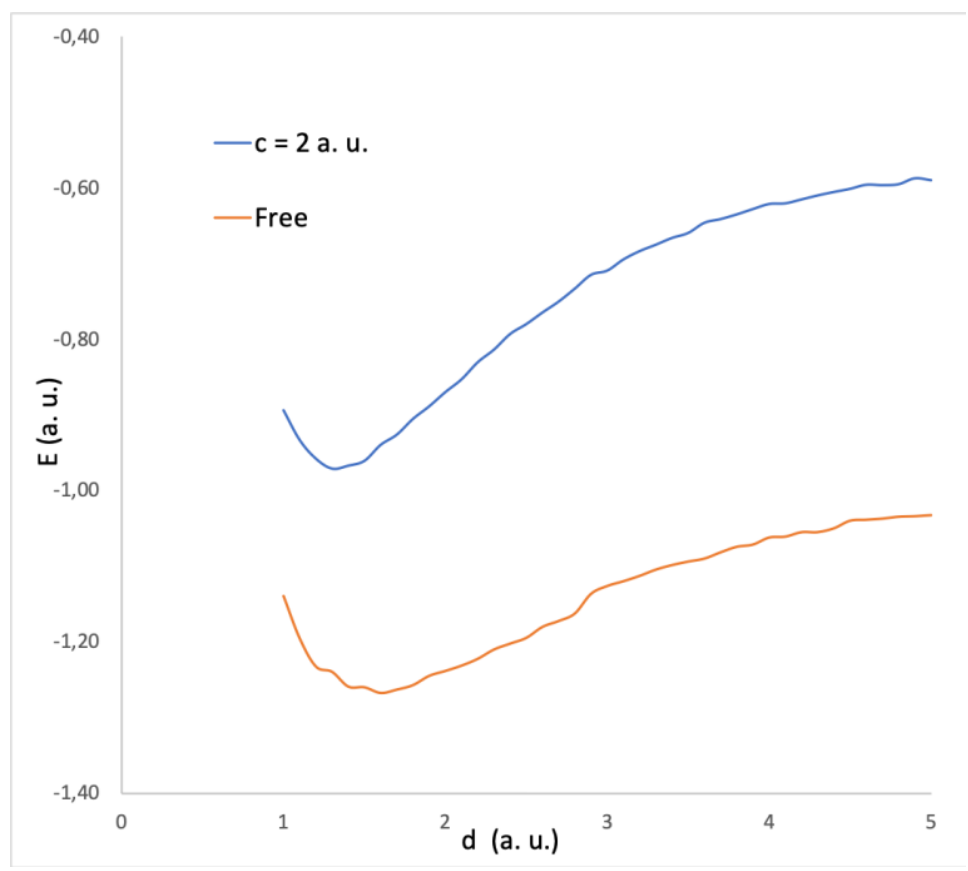

Figure 3. Potential energy curves of a free collinear $\mathrm{H}_{3}{ }^{+}$and of a collinear $\mathrm{H}_{3}{ }^{+}$confined in a nanotube with confinement dimension $c=2$ a. u. .

The universal features of the stochastic description manifest themselves here, not only because of the easy adaptability of the code to different quantum problems, but also in view of the adaptability of a code based on the diffusion equation to completely different problems. For example, DMC was applied to the description of the dissociation of $\mathrm{CO}_{2}$ molecules in the context of greenhouse effect mitigation [Diomede, 2017]. Despite being developed for a classical problem of chemical kinetics, the last code is essentially the same as a DMC code for solving the quantum problem: same mathematical foundations (but different interpretations of symbols), code structure, management of random events. This universality is a very concrete advantage as to multidisciplinary code development.

\section{Models of the kinetic and thermal behavior of nanoparticles in low temperature plasma}

In plasma reactors, nanoparticles of various compositions can be produced as a result of chemical reactions. Arguably, the most important mechanism governing the dynamics of the nanoparticles in dusty plasma is particle charging. The nanoparticles inside plasma acquire a charge due to frequent collisions with the charged species, ions and electrons. In fact, most of the particle dynamics of the nanoparticles such as formation, growth, temperature and transport are strongly influenced by their charging process that is governed by the ion and electron current at the nanoparticle. 
Having an analytical expression for the current at a solid nanoparticle is difficult for most cases and molecular dynamics Monte Carlo simulations where the motion of charged particles is integrated using a velocity Verlet algorithm is often used to study the charging of particles. The motion of particles is well described by a Langevin equation with the effects of collisions represented by a statistical Gaussian source term [Gatti, 2008; Gopalakrishnan, 2012; Chahl, 2019] with inclusion of various other forces.

The simplest theory for the charging of solid particle immersed in plasma is the orbital motion limited theory (OML) [Allen, 1992] which is valid for most low pressure. The theory highlights that an ion of a given kinetic energy entering a limiting orbit would be eventually captured by the particle. The dimension of the limiting orbit is generally small compared to the mean free path and collisions can be assumed to be non-existent in this small region. In this case, the electron and ion currents can be derived from the straightforward expressions based on Orbit Motion Limited theory (OML) for nocollisional sheath [Allen, 1992]:

$$
\begin{gathered}
j_{e}=n_{e}\left(\frac{k_{b} T_{e}}{2 \pi m_{e}}\right)^{1 / 2}\left(1-\exp \left(\frac{e \varphi}{k_{b} T_{e}}\right)\right) \\
j_{i}=n_{i}\left(\frac{k_{b} T_{g}}{2 \pi m_{i}}\right)^{1 / 2}\left(1-\frac{e \varphi}{k_{b} T_{g}}\right)
\end{gathered}
$$

Where $j_{e} / j_{i}, n_{e} / n_{i}$ and $m_{e} / m_{i}$ are the electron/ion flux densities, and masses, respectively. $\phi$ is the electrical potential of the particle with respect to the surrounding plasma, and $k_{b}$ is the Boltzmann constant. The heavy species, i.e., neutrals and ions, are assumed to be in thermal equilibrium at a temperature $T_{g}$, while the electrons are assumed to be at a thermal non-equilibrium with usually a much higher temperature $T_{e}$. Actually, the RHS of equation (4) represents the thermal flux of electrons that can overcome the potential energy barrier imposed by the negative charge of the particle, i. e. only those electrons with a thermal kinetic energy that is above the potential barrier can reach the particles. The RHS of equation (5) represents the population of ions that reach the particles estimated on the basis that an ion dynamic in the vicinity of the particle is governed by the central coulomb potential field (no collision) and that only those ions with an initial velocity that lead to a trajectory that fall on the particle contribute to the ion flux on the particle.

At steady state, the electrical current collected by a particle immerged in a plasma is zero, i.e. the particle is at the floating potential. The particle charge and potential can be estimated by expressing the balance between electron and ion currents as inferred from equations (4) and (5) and assuming that the 
particles behave like spherical capacitance, i. e., $\varphi=\frac{q_{p}}{4 \pi \varepsilon_{0}} \frac{1}{r_{p}}$, with $q_{p}$ and $r_{p}$ the charge and radius of the particle.

However, the OML approximation is not valid for various conditions encountered in plasma [Goree, 1994]. For example, at moderate to high pressures, the mean free path decreases and ions and electrons may undergo frequent collisions with other neutral species inside the sheath of the particle. The frequent collisions can reduce the kinetic energy of the charged particles and can be eventually be captured by the particles. In other words, the presence of collisions can enhance the ion and electron currents at the particle surface. Furthermore, there can be other effects such as image potentials which can further alter the current of charged particles. There has been extensive work to improve the charging model incorporating different effects in the literature [Allen, 1992; Khrapak, 2006; D’yachkov, 2007; Gatti, 2008; Gopalakrishnan, 2012; Chahl, 2019].

When the charging process may be assumed continuous, the average charge carried by a solid particle is such that there is a balance between negative and positive currents at the particle. In a typical dusty plasma environment, the particles acquire a net negative charge owing to the higher electron mobility. Further, due to the difference between the ion and electron mobilities, the particle charge is subjected to fluctuations. In fact, description of charge fluctuations has been identified to critical for particle growth and morphology. [Cui, 1994] performed Monte Carlo simulation of particle charging and showed that charge fluctuations can be very significant. Further, the particle charging can be transformed into a Fokker-Planck description to obtain the mean particle charge and distribution [Matsoukas, 1995; Matsoukas, 1997]. It was shown that, for OML theory of particle charging, the particle charge distribution can be represented as a Gaussian [Matsoukas, 1995; Matsoukas 1997]. However, it has to be noted that charge is discrete and modeling particle charging using a continuous model may not accurately describe the charging process [Michau, 2019]. This is especially the case when the charge fluctuation is of the same order as the average charge, which usually takes place for small solid particles, i.e., typically less than $10 \mathrm{~nm}$. In this case, we consider the stochastic nature of particle charging as a Poisson's process where the charge distribution is represented as a set of integer charge states and the charge distribution is obtained by solving the master equations (equation 1) for charge fraction $\varphi_{q}$ of each charge state $q$ given by:

$$
\frac{1}{S_{p}} \frac{d \varphi_{q}}{d t}=j_{e, q+1} \varphi_{q+1}+j_{i, q-1} \varphi_{q-1}-\left(j_{e, q}+j_{i, q}\right) \varphi_{q}
$$

Where $j_{e, q}$ and $j_{i, q}$ are the ion and electron currents respectively, and $S_{p}$ is the surface area of the particle. The solution of the previous equation requires a current law that may be driven from OML or any other theory. 
The difference between the continuous model and the discrete model of particle charging is very stark for small particles as seen in the tabulations of average charge of particles using the two models.

\begin{tabular}{|c|c|c|}
\hline $\mathbf{r}_{\mathbf{p}}(\mathbf{n m})$ & $\begin{array}{c}\mathbf{q}_{\text {ref }} \text { (number of } \\
\text { charges) }\end{array}$ & $\begin{array}{c}\mathbf{q}_{\text {stat }} \text { (number of } \\
\text { charges) }\end{array}$ \\
\hline 1 & -0.25 & -0.91 \\
\hline 2.5 & -0.63 & -0.99 \\
\hline 5 & -1.26 & -1.66 \\
\hline 50 & -12.6 & -12.9 \\
\hline
\end{tabular}

Table 1. Average charge of particles calculated using OML equilibrium and statistical approach for $n_{e}=n_{i} . q_{r e f}$ is the reference particle charge calculated using the OML theory with the electron and ion current given by equations (4) and (5). $q_{\text {stat }}$ is the particle charge derived from the statistical model (equation (6)).
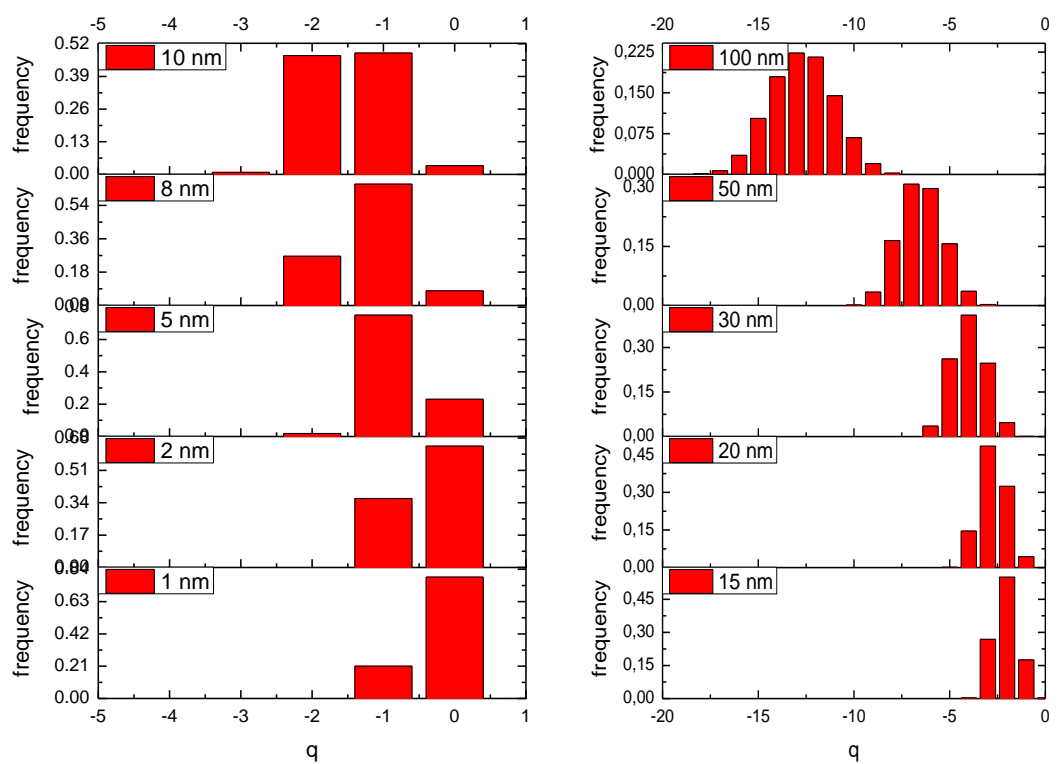

Figure 4: Particle charge distributions obtained for different particles sizes in a plasma described in [Michau, 2020] with electron density $10^{11} \mathrm{~cm}^{-3}$ and electron temperature of $0.1 \mathrm{eV}$.

Figure 4 shows the charge distribution for nanoparticles of different sizes obtained using Monte Carlo simulations inside a plasma as described in [Michau, 2019]. While the Gaussian approximation for 
charge distribution was appropriate for large particles, such an approximation is no more correct for very small particles.

One of the side-effects of charge fluctuation is the stochastic thermal balance of the particle. Due to continuous charging, the average particle temperature can be significantly higher than the surrounding gases [Swinkels, 2000; Arnas, 2009; Maurer, 2011]. Further it was shown in [Prasanna, 2019] that the average particle temperature calculated using a continuous theory [Maurer, 2011] would severely under predict the temperature averaged from Monte Carlo simulation (Table 2). This is mainly because the stochastic charging process also results in a stochastic heating which is not accounted by the continuous theory. This is even more significant for small particles.

\begin{tabular}{|c|c|c|}
\hline $\mathbf{r}_{\mathbf{p}}(\mathbf{n m})$ & $\begin{array}{c}\mathbf{T}(\mathbf{K}) \\
\text { Continuous }\end{array}$ & $\begin{array}{c}\text { T }(\mathbf{K}) \text { Monte } \\
\text { Carlo }\end{array}$ \\
\hline 1 & 465 & 709 \\
\hline 2.5 & 465 & 518 \\
\hline 5 & 465 & 490 \\
\hline 50 & 465 & 468 \\
\hline
\end{tabular}

Table 2. Average particle temperature using continuous model and Monte-carlo simulations for the plasma conditions described in [Michau, 2019].

In order to accurately describe the stochastic nature of charging and thermal balance, a population balance equation for charge $q$ and temperature $T_{p}$ was developed by [Prasanna, 2019]. The solution of the population balance equations permitted to obtain both the charge and temperature distribution functions for the particles under different plasma conditions. Figure 5 shows the normalized particle cumulative temperature distribution (NCPTD) obtained for a particle size of $1 \mathrm{~nm}$ radius for the plasma described in [Michau, 2019] for different electron densities. The value of the NPCTD at a given temperature $T$ corresponds to the fraction of particles with temperature higher than $T$. It is remarkable that one can find substantial quantities of particles at high temperatures. As a matter of fact, $10 \%$ of the particle shows temperature-balues above $1000 \mathrm{~K}$ and $1 \%$ of the partuicles have a temperatire abve 2000 $\mathrm{K}$ for an electron density of $10^{11} \mathrm{~cm}^{-3}$. The fraction of particles having high temperature decreases with the electron densities. The electron densities of the plasma depletes under strong presence of dusts. The results are significant for plasma processing of crystalline nanoparticles. The critical temperatures for obtaining crystalline nanoparticles is a function of particle radius [Hirasawa, 2006] and the possibility of having large fractions of particles at higher temperature permits having higher yield of crystalline particles. In that sense, one needs to design a plasma process such that dilute quantities of particles 
nucleate in the plasma so that particles can easily reach temperatures greater than crystallization temperature [Mangolini, 2009; Kramer, 2015].

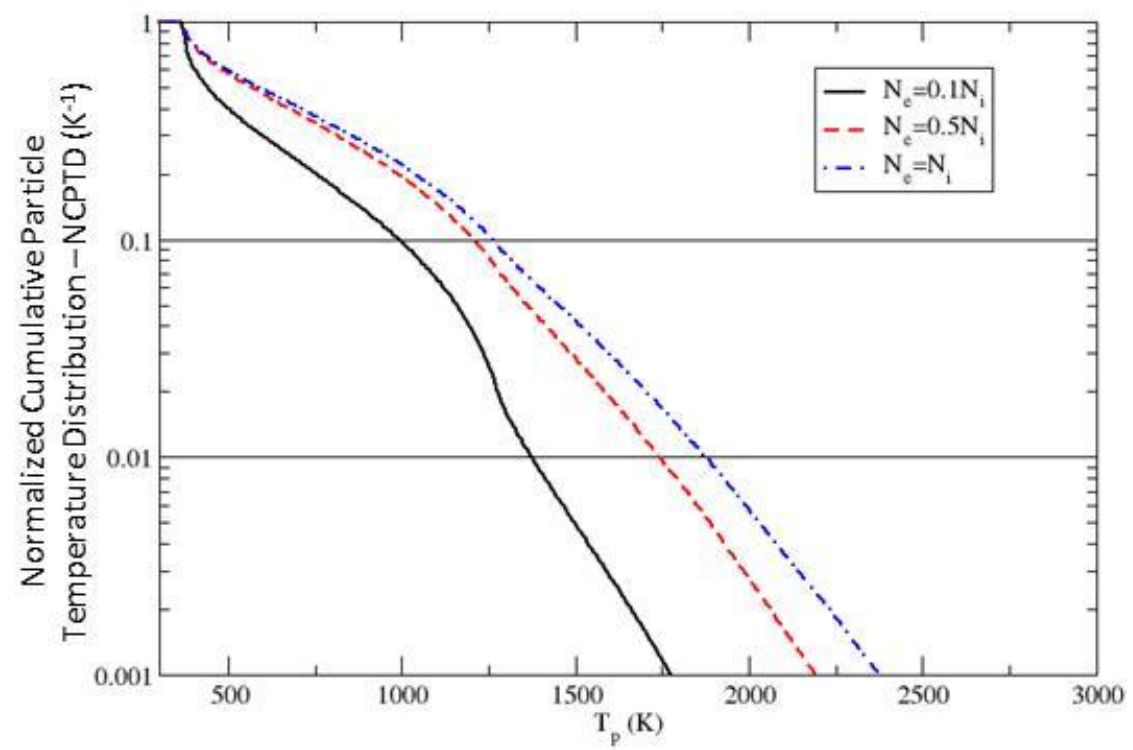

Figure 5. Cumulative particle temperature distribution functions for particle size of $1 \mathrm{~nm}$ for the plasma conditions described in [Michau, 2019] for ion densities fixed at $10^{11} \mathrm{~cm}^{-3}$ and different electron densities corresponding to different discharge electronegativity.

The other direct consequence of charge fluctuation is growth of particles through coagulation. As most particles are negatively charged, growth of nanoparticles by coagulation is very limited. However, small particles can be neutrally charged due to charge fluctuations (as seen in Figure 5) and there can be coagulation between large negatively charged particles and small neutral or positively charged particles. The importance of charging model discussed earlier is critical in order to obtain a good estimate of the coagulation rates [Le Picard, 2016; Mamunuru, 2017]. Like particle charging, coagulation rates between two particles depends on multitude of parameters such as mean free path (free regime, transition regime or continuous regime). Further, the attractive forces between the charged particles can increase due to polarization [Huang, 1991; Mankelevich, 2002; Mankelevich, 2008]. The velocity Verlet algorithm can also be employed to determine the coagulation rates for different conditions [Gopalakrishnan, 2012].

Finally, the different statistical models described to determine the dynamics of the particles act as inputs to fluid aerosol models which can capture the big picture of the dusty plasma dynamics and determine the space-time distribution of particle density, mean particle diameter and particle size distribution. Several fluid aerosol dynamic studies [Ravi, 2009; Agarwal, 2012; Michau, 2017; Michau, 2019] have stressed the importance of charging models and its other effects discussed in the paper on having a simulation comparable with the experiments. 


\section{Models of neutral transport in plasma reactors}

Low pressure reactors (0.1-100 millitor range), possibly associated with electrical ionization of the residual gas (plasma reactors), have unique characteristics in the production of nanomaterials and functionalized thin films [Chen, 2012; Kashtanov, 2007; Meyyappan, 2011; Bianco, 2018], with the possibility to obtain excellent uniformity and reproducibility and much reduced heating of substrate and the film, avoiding thermal reactions that can spoil the final result. Computer modeling allows to obtain useful information on the phenomena occurring in reactors at the macroscopic scale, provides insight for their optimal use avoiding a complex diagnostic, and allows to estimate the effects of geometry changes without actually performing very expensive modification of the real reactor. In this respect, the use of stochastic methodology allows to solve the most complete forms of the transport equation avoiding the use of approximations which are often non tenable in the extreme non equilibrium conditions found in these devices [Longo, 2004; Longo, 2006; Donkó, 2011a].

In the past, our research groups produced several simulation codes for to the prediction of the chemical reactions of transport phenomena and collective phenomena on a macroscopic scale that occur in plasma reactors, with both capacitive and inductive coupling to the electric generator [Hassouni, 1999; Capitelli, 2002; Diomede, 2007; Diomede, 2008].

An example of application where a multiscale approach was adopted is the production of Cs/Mo bimetallic surfaces as catalyst in the Neutral Drive concept for the ITER nuclear fusion experiment [Dudnikov, 1992; Diomede, 2009, Taccogna 2008]. The macroscopic models for this system, employing Monte Carlo models of the plasma phase and transport processes, were also supported by rigorous ab initio simulations by methods of quantum chemistry [Damone, 2015], in which Cs atoms were launched on a pristine 001 Mo substrate, which allowed to study the partially ordered geometry of a molybdenum surface covered by half a cesium monolayer. This appears to be the most efficient catalyst system for this application, thereby suggesting that the "random nanostructure" of a partial Cs coverage, leaving part of the substrate metal visible to hydrogen molecules, is beneficial to get the highest reaction yield. Our set of nuclear coordinates for Cs-covered Mo (Figure 6), which is available on request to the authors, has been used in several subsequent calculations by other groups, e.g. [Rutigliano, 2017]. 


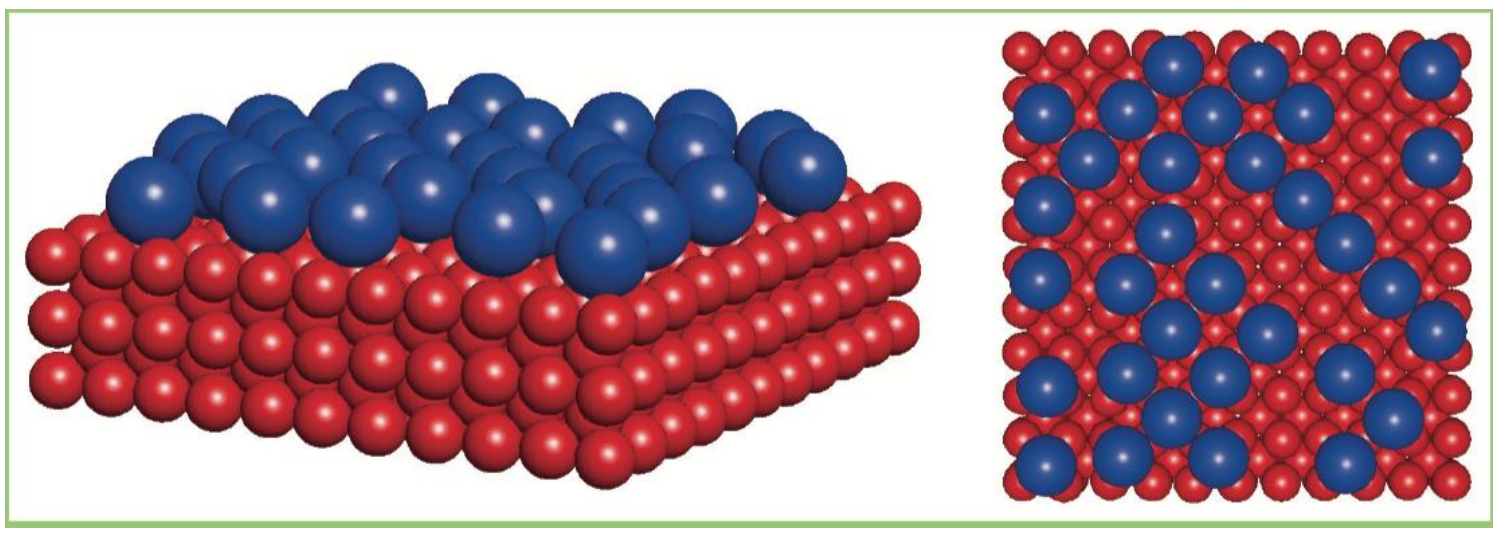

Figure 6. The geometry calculated for a half-monolayer of cesium on molybdenum showing the wide range of "random nanofeatures" arising from the ballistic deposition and diffusion process. Cover image of Plasma physics and controlled Fusion vol.57, no.3, March 2015 [Damone, 2015].

At the macroscopic scale, as mentioned, the use of stochastic methodology in the study of the transport of neutral particles in the context of plasma reactors is an extremely important topic, since these chemically activated particles from the plasma (for example, in the form of radicals or excited species) can start high added value chemical reactions that take place on the substrate, while avoiding heating.

Recently, our activity has focused on the development of models for the transport of neutral particles based on the stochastic solution of the transport equation in integral form [Longo, 2006; Longo, 2009; Galtier, 2013]. The method can be defined as a variant of the Ray Tracing technique used in computer graphics: the primary source of neutral particles is assimilated to a source of radiation that produces a large number of rays (from a few thousands to several millions), which simulate both the scattering by inner reactor surfaces and by ambient gas molecules, thanks to a Monte Carlo random selection technique.

A very interesting feature of this approach is the possibility of simply describing, within a handcrafted program, real geometry of even very complex reactors, thus allowing a unique capacity for customization and experimentation, as well as the possibility of adding, with relative ease, additional elements of reactor physics and chemistry, which is often not allowed by commercial programs. In this way the program GAS was recently created in a Fortran90 version, while a Python3 version is being developed. The program uses a space description matrix (Figure 7, left) to reduce strongly the cost of the detection of boundaries. The numerical value associated with each element of this matrix corresponds to a volume occupied by gas or to a solid structure, for which it is also possible to specify the physical properties: for example, the structure can adsorb the traced particles or diffuse them with an isotropic or more complex law. The same matrix is used to sample the particles during the motion and produce a density plot (Figure 7, right). In this way, it is possible to describe reactors with any geometry: an example is shown in figure 8. In order to show the possibilities offered by this type of simulation, figure 9 illustrates a toy system that can be called "maze reactor": this system should not be 
taken seriously as a geometry in a reactor for the treatment of materials, but it is a demonstration of the achievable geometric complexity with simple instructions of this type, based on the geometric definition of the values of matrix elements.
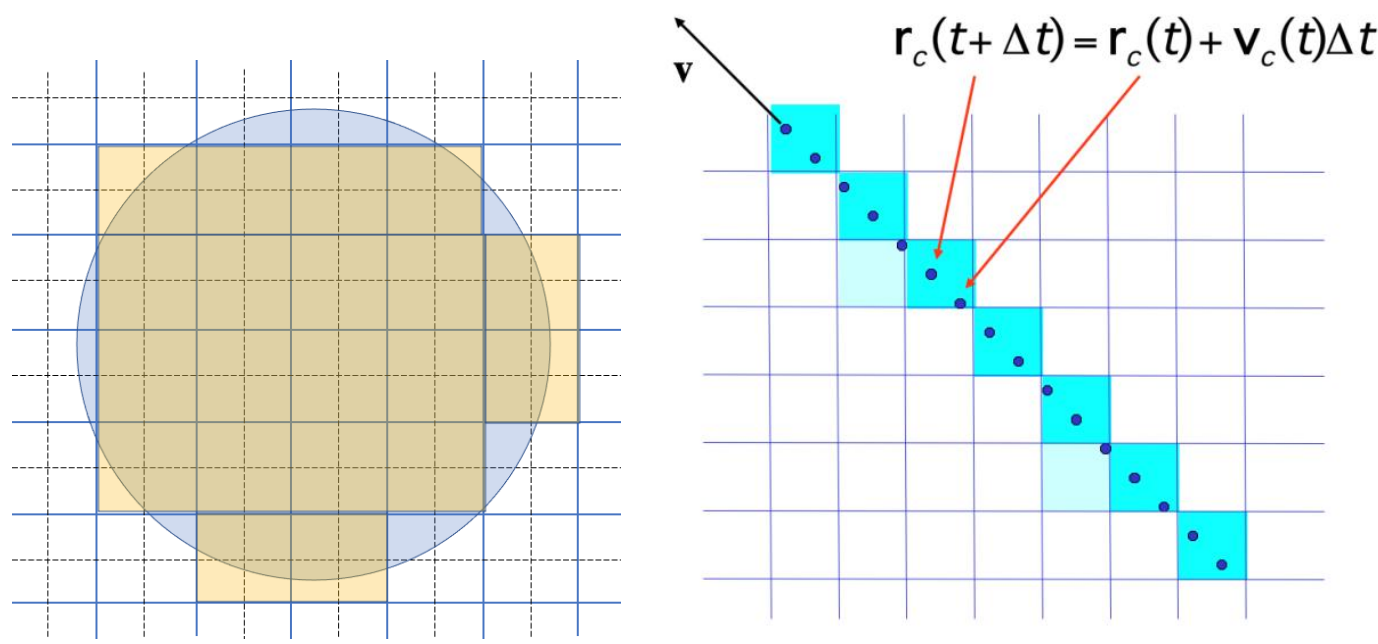

Figure 7. Uses of the mesh in the GAS model. Left: in this example, the marked cells are used to locate the surface of a cylindrical object as part of a reactor model (the resolution is much better in actual calculations). The figure shows that, while the centers of the mesh cells are scanned, they are compared with the analytical expression of the feature: any cell whose center is included into the feature is marked by a special value of a flag (represented by the yellow color). The simulated particles which enter into such marked cells change their status, in order to describe their interaction with the feature surface. The visible irregularities of the stored shape obviously disappear with a denser mesh. Right: storing particle data on the same matrix to produce a density plot. 


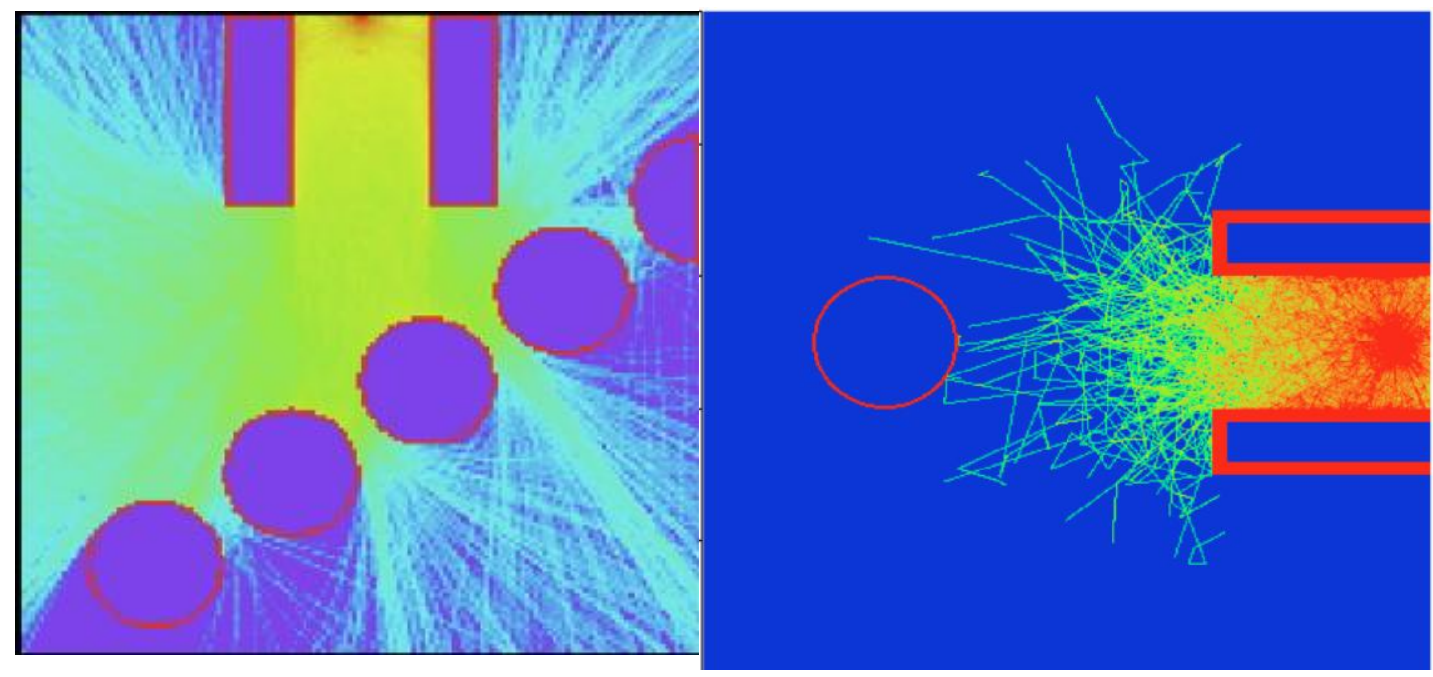

Figure 8. Left: Ray Tracing (RT) in vacuum with scattering surfaces of different shapes. The particles are injected from center-top. The figure is a 2D slice of a 3D geometry infinitely extended vertically: therefore, the two obstacles in the upper region are prisms, the 5 obstacles are cylinders. All surfaces diffuse incident particles isotropically. Right: time-dependent transport of neutral particles, including scattering from the bulk gas, in a model reactor using the GAS code. Two prisms and a cylinder are introduced as isotropic diffusing obstacles.

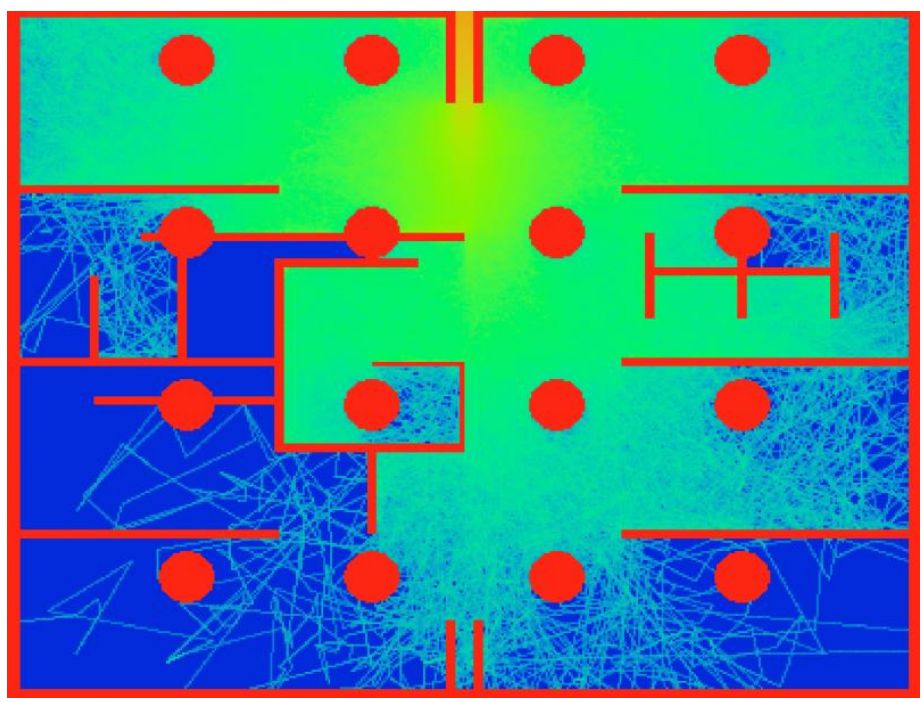

Figure 9. The "maze reactor": a test geometry for the GAS code created by Ms Brunella D'Anzi, Master student in Physics.

The traced particles are injected into the bulk gas from the inlet above. Conventions are the same as in Figure 8. 
It is possible to simulate the transport and diffusion of particles introduced from a source into a point of the reactor in a simple way, and obtain any type of potentially measurable quantity in a reactor of this type, such as the collision frequency (hits per unit surface per second) on any of the walls and the average concentration of particles of the species introduced into the volume of the gas in each point of the gas phase.

In the past, we included in models much more simplified geometry, some refined aspects of transport physics: for example, the thermalization of high-energy radicals produced by exothermic reactions [Panarese, 2013] and the transport of atomic particles in transonic and viscous gas flows (including shocks), calculated in turn by non-linear stochastic techniques for the same reactor geometry [Longo, 2009]. These routines are now going rewritten to be included in the GAS code. Thus, we will be able soon to study systems characterized by both chemical-physical and geometric complexity at the macroscopic level.

Even if in the demonstrations here the reactor geometry is two-dimensional, the motion and scattering of the particles is three-dimensional, and there is no difficulty, neither in principle nor in practice, in simulating complex three-dimensional systems using the same method, apart the increased computational cost and some obvious issues of visual rendering.

The setting of the neutrals transport problem as a ray tracing problem also has another advantage: it is possible, and it was demonstrated in a recent publication [Longo, 2019], to take advantage of the formal analogy between the transport problem of neutral particles and Radiative Transfer (RT) theory to reformulate, at least under certain symmetry conditions, the transport problem in a particularly convenient integral form, i.e. a generalization of the Schwarzschild-Milne equation, which include terms describing the chemical reactions in the gas phase and the catalytic properties of the reactor wall. These deterministic methods, which also arise from the theory of stochastic models, allow to obtain considerable accelerations in terms of calculation time with respect to the corresponding Monte Carlo formulation and allow an easier integration of the corresponding module with deterministic multiphysical models of a full reactor.

\section{Conclusions}

As can be seen from the study cases presented in this work, the computer simulation based on the ideas of the stochastic process theory concretely allows to study in a quantitative way phenomena and objects in the realms of nanoscience, ranging from the microscopic scale of the smallest confined molecules and nanoparticles to the macroscopic scale of plasma reactors or vacuum systems, used in the production of nanostructured surfaces. It also allows to examine very different aspects of the physics of these systems at their respective scales, ranging from the response to static potentials to the dynamic 
response to strong non-equilibrium or time-dependent conditions. The universality of the formalism allows to develop custom and native codes, starting from algorithm and sources originally developed for very distant and different applications. Many years of experience in this direction taught us that when, as in our case, there are already considerable computer programming skills in the collaboration, the creation of native codes exploiting these universal features allows to get the highest versatility, prompt answers to current research issues, and also provides a unique first-hand experience in understanding the physical principles.

\section{Bibiography}

Agarwal, P., \& Girshick, S. L. (2012) Plasma Sources Science and Technology, 21(5) 055023.

Allen, J. E. (1992) Physica Scripta, 45(5) 497.

Arnas, C., \& Mouberi, A. A. (2009) Journal of Applied Physics, 105(6) 063301.

Bianco, G. V., Sacchetti, A., Ingrosso, C., Giangregorio, M. M., Losurdo, M., Capezzuto, P., \& Bruno, G. (2018) Carbon, 129 869-877.

Capitelli, M., Celiberto, R., Esposito, F., Laricchiuta, A., Hassouni, K., \& Longo, S. (2002) Plasma Sources Science and Technology, 11(3A) A7-A25.

Chahl, H. S., \& Gopalakrishnan, R. (2019) Aerosol Science and Technology, 53(8) 933-957.

Chen, Francis F., and Jane P. Chang. Lecture notes on principles of plasma processing. Springer Science \& Business Media, 2012.

Chuu, D.S., Hsiao, C.M., \& Mei, W.N. (1992) Physical Review B 46, 3898.

Colín-Rodríguez, R., Díaz-García, C., \& Cruz, S.A. (2011) Journal of Physics B: Atomic, Molecular and Optical Physics, 44(24) 241001.

Cui, C., \& Goree, J. (1994) IEEE Transactions on plasma science, 22(2) 151-158.

Damone, A., et al. (2015) Plasma Physics and Controlled Fusion 57(3) 035005.

Diomede, P., Hassouni, K., Longo, S., \& Capitelli, M. (2007) IEEE transactions on plasma science, 35(5) 1241-1246.

Diomede, P., Michau, A., Redolfi, M., Morscheidt, W., Hassouni, K., Longo, S., \& Capitelli, M. (2008) Physics of Plasmas, 15(10) 103505. 
Diomede, P., \& Longo, S. (2009) Plasma Sources Science and Technology, 19(1) 015019.

Diomede, P., Van De Sanden, M. C., \& Longo, S. (2017). The Journal of Physical Chemistry C, 121(36), 19568-19576.

Donkó, Z. (2011a). Plasma Sources Science and Technology, 20(2) 024001.

Dudnikov, V. G. (1992). 20 years of cesium catalysis for negative ion production in gas discharges. Review of scientific instruments, 63(4), 2660-2668.

D’yachkov, L. G., Khrapak, A. G., Khrapak, S. A., \& Morfill, G. E. (2007) Physics of plasmas, 14(4) 042102.

Foulkes, W. M. C., Mitas, L., Needs, R. J., \& Rajagopal, G. (2001) Reviews of Modern Physics, 73(1) 33.

Galtier, M., Blanco, S., Caliot, C., Coustet, C., Dauchet, J., El Hafi, M., ... \& Piaud, B. (2013). Journal of Quantitative Spectroscopy and Radiative Transfer, 125 57-68.

Gatti, M., \& Kortshagen, U. (2008) Physical Review E, 78(4) 046402.

Gopalakrishnan, R., \& Hogan Jr, C. J. (2012) Physical Review E, 85(2) 026410.

Goree, J. (1994) Plasma Sources Science and Technology, 3(3) 400.

Harrison, P. (2005) Quantum Wells, Wires and Dots: Theoretical and Computational Physics of Semiconductors Nanostructures, Wiley-Interscience, New York.

Hassouni, K., Grotjohn, T.A., \& Gicquel, A. (1999) Journal of Applied Physics, 86(1) 134-151.

Hirasawa, M., Orii, T., \& Seto, T. (2006) Applied physics letters, 88(9) 093119.

Huang, D. D., Seinfeld, J. H., \& Okuyama, K. (1991) Journal of colloid and interface science, 141(1) 191-198.

Kashtanov, P. V., Smirnov, B. M., \& Hippler, R. (2007) Physics-Uspekhi, 50(5) 455.

Khrapak, S. A., Morfill, G. E., Khrapak, A. G., \& D’yachkov, L. G. (2006) Physics of plasmas, 13(5) 052114.

Kramer, N. J., Aydil, E. S., \& Kortshagen, U. R. (2015) Journal of Physics D: Applied Physics, 48(3) 035205 .

Le Picard, R., \& Girshick, S. L. (2016) Journal of Physics D: Applied Physics, 49(9) 095201.

Longo, S., \& Diomede, P. (2004) The European Physical Journal Applied Physics, 26(3) 177-185.

Longo, S. (2006) Plasma Sources Science and Technology, 15(4) S181. 
Longo, S., \& Diomede, P. (2009) Journal of Computational Physics, 228(10) 3851-3857.

Longo, S., Micca Longo, G., \& Giordano, D. (2018) Rendiconti Lincei. Scienze Fisiche e Naturali, 29 $173-177$.

Longo, S., Micca Longo, G., Pavone, E., \& Schiavone, F. (2019) Plasma Sources Science and Technology, 28(12), 125008.

Mamunuru, M., Le Picard, R., Sakiyama, Y., \& Girshick, S. L. (2017) Plasma Chemistry and Plasma Processing, 37(3) 701-715.

Mangolini, L., \& Kortshagen, U. (2009) Physical review E, 79(2) 026405.

Mankelevich, Y. A., Olevanov, M. A., \& Rakhimova, T. V. (2002) Journal of Experimental and Theoretical Physics, 94(6) 1106-1113.

Mankelevich, Y. A., Olevanov, M. A., \& Rakhimova, T. V. (2008) Plasma Sources Science and Technology, 17(1) 015013.

Matsoukas, T., \& Russell, M. (1995) Journal of applied physics, 77(9) 4285-4292.

Matsoukas, T., \& Russell, M. (1997) Physical Review E, 55(1) 991.

Maurer, H. R., \& Kersten, H. (2011) Journal of Physics D: Applied Physics, 44(17) 174029.

Meyyappan, M. (2011) Journal of Physics D: Applied Physics, 44(17) 174002.

Micca Longo, G., Longo, S., \& Giordano, D., (2015a) Physica Scripta, 90025403.

Micca Longo, G., Longo, S., \& Giordano, D., (2015b) Plasma Sources Science and Technology, 24 065019 .

Micca Longo, G., Longo, S., \& Giordano, D., (2015c) Physica Scripta, 90085402.

Michau, A., Swaminathan, P., Longo, S., \& Hassouni, K. (2019) Plasma Physics and Controlled Fusion, 62(1) 014002.

Oliveira Batael, Hugo, and Elso Drigo Filho. Theoretical Chemistry Accounts139.8 (2020): 1-7.

Panarese, A., Diomede, P., \& Longo, S. (2013) Plasma Sources Science and Technology, 22(4) 045017.

Prasanna, S., Michau, A., Hassouni, K., \& Longo, S. (2019) Plasma Sources Science and Technology, 28(3) 03LT03.

Ravi, L., \& Girshick, S. L. (2009) Physical Review E, 79(2) 026408.

Rutigliano, M., Palma, A., \& Sanna, N. (2017) Surface Science, 664 194-200. 
Sabin, J.R., Brändas, E., \& Cruz, S.A. (2009) The Theory of Confined Quantum Systems, Part I and II, Advances in Quantum Chemistry, Vols. 57 and 58, Academic Press, Cambridge, Massachusetts.

Sarsa, A., \& Le Sech, C. (2012) Journal of Physics B: Atomic, Molecular and Optical Physics, 45(20) 205101.

Sen, K. D., (Eds.). (2014). Electronic structure of quantum confined atoms and molecules. Switzerland: Springer International Publishing.

Swinkels, G. H. P. M., Kersten, H., Deutsch, H., \& Kroesen, G. M. W. (2000) Journal of applied physics, 88(4) 1747-1755.

Taccogna, F., Schneider, R., Longo, S., \& Capitelli, M. (2008). Physics of Plasmas, 15(10), 103502. 